\title{
Integrated mutation, copy number and expression profiling in resectable non-small cell lung cancer
}

\author{
Genni M Newnham ${ }^{1, *^{*}}$, Matthew Conron², SueAnne McLachlan ${ }^{1,8}$, Alexander Dobrovic ${ }^{3,9}$, Hongdo Do ${ }^{3,9}$, Jason Li ${ }^{5}$, \\ Kenneth Opeskin ${ }^{4}$, Natalie Thompson ${ }^{5}$, Gavin M Wright ${ }^{6}$, David M Thomas $^{7}$
}

\begin{abstract}
Background: The aim of this study was to identify critical genes involved in non-small cell lung cancer (NSCLC) pathogenesis that may lead to a more complete understanding of this disease and identify novel molecular targets for use in the development of more effective therapies.

Methods: Both transcriptional and genomic profiling were performed on 69 resected NSCLC specimens and results correlated with mutational analyses and clinical data to identify genetic alterations associated with groups of interest.

Results: Combined analyses identified specific patterns of genetic alteration associated with adenocarcinoma vs. squamous differentiation; KRAS mutation; TP53 mutation, metastatic potential and disease recurrence and survival. Amplification of $3 q$ was associated with mutations in TP53 in adenocarcinoma. A prognostic signature for disease recurrence, reflecting KRAS pathway activation, was validated in an independent test set.
\end{abstract}

Conclusions: These results may provide the first steps in identifying new predictive biomarkers and targets for novel therapies, thus improving outcomes for patients with this deadly disease.

\section{Background}

Non-small cell lung cancer (NSCLC) is the commonest cause of cancer death in Western communities. Current treatments offer the potential of cure only to the small number of patients who present with early stage NSCLC, whilst outcomes for those with advanced disease remain poor. Recent advances including adjuvant chemotherapy and targeted biological therapies have lead to modest improvements in survival for small subgroups of patients. Clearly new treatment approaches are required to substantially improve outcome. As has been the case for other tumour types, molecular profiling techniques have the potential to provide benefit through improved understanding of disease pathogenesis, identification of subgroups in whom current therapies are most likely to be effective and in the development of novel therapies.

\footnotetext{
* Correspondence: Genni.Newnham@svhm.org.au

'Department of Oncology, St Vincent's Hospital, (Victoria Pde), Melbourne, (3065), Australia

Full list of author information is available at the end of the article
}

Genetic heterogeneity is a feature of NSCLC, with varying combinations of multiple molecular alterations contributing to tumour development [1]. A key challenge for high-throughput molecular profiling techniques is to distinguish between genes whose expression is altered directly by heritable changes in gene function and those where changes are an inevitable down-stream consequence of primary changes to genes directly involved in disease pathogenesis. Correlation of transcriptional and genomic data allows more focussed analysis of the large number of genetic alterations identified by molecular profiling. This study incorporates the results of both transcriptional and genomic profiling for clinically relevant subgroups of NSCLC to identify genes of potential predictive or pathogenic importance in this deadly disease.

\section{Methods \\ Samples}

After obtaining institutional ethics approval, patients with stage I-IIIA NSCLC seen by the St Vincent's Hospital
C Biomed Central

(c) 2011 Newnham et al; licensee BioMed Central Ltd. This is an Open Access article distributed under the terms of the Creative Commons Attribution License (http://creativecommons.org/licenses/by/2.0), which permits unrestricted use, distribution, and reproduction in any medium, provided the original work is properly cited. 
Combined Lung Service between February 2004 and July 2006 and planned for curative resection were invited to participate. Exclusion criteria included age $<18$ years, administration of neoadjuvant chemotherapy, and inability to provide informed consent. Integrated demographic, radiological, pathological and outcome data was collected for all consenting patients.

In addition, a small number of samples collected earlier and stored in the Peter MacCallum (PeterMac) tissue bank were utilised after approval by the PeterMac Tissue Management Committee.

\section{Microarray analyses}

Samples of tumour $\left(\geq 1 \mathrm{~cm}^{3}\right)$ were selected from fresh specimens, and then stored whole at $-180^{\circ} \mathrm{C}$. Only those specimens containing $>75 \%$ tumour cells and $<25 \%$ necrosis were used in molecular studies. Both RNA and DNA were isolated from each sample for analysis using established protocols (see additional files 1 and 2). Transcriptional profiling was performed using 10,500 element cDNA microarrays (PeterMac, Melbourne, Australia) [2]. Genomic profiling using 2400 element bacterial artificial chromosome (BAC) arrays was completed at the University of San Francisco, California, USA [3]. Detailed description of transcriptional and genomic profiling is included in additional files 3 and 4 .

\section{Mutation analyses}

All samples were tested for TP53 mutations and all adenocarcinoma (AC) and large cell carcinoma (LCC) samples were screened for KRAS mutations using high resolution melting analysis $[4,5]$ with or without DNA sequencing.

\section{Bioinformatic analyses}

The effect of histology, presence or absence of $K R A S$ or TP53 mutation, tumour size and metastasis status, recurrence within 12 months of surgery and survival on gene expression was explored. After removing control genes, analysis was conducted in CRAN, R Bioconductor using the LIMMA [6,7] package to generate detailed lists of gene expression differences with significance $\mathrm{p}$ values between each subgroup of interest. To account for multiple testing, $p$ values of $<0.005$ were considered statistically significant. Gene lists were then interrogated using publicly available programs (Intelligent Systems and Bioinformatics Laboratory, Wayne State University, Detroit, MI, USA, http://vortex.cs.wayne.edu/projects.htm) to identify gene ontology and molecular pathway patterns.

Changes in the normalized and smoothed genomic data [8] were assigned stepwise copy change levels from -2 to $3(-2=$ homozygous loss, $-1=$ heterozygous loss, 1 = single copy gain, 2 = gain of two copies, 3 = highlevel gain, $0=$ normal copy number). Using these standardised copy number values, it was possible to make comparisons of the frequency of each level of copy change at each BAC location for specific groups of interest.

To compare our data with that of other groups of patients with early stage NSCLC, we performed comparisons with publicly available external data sets obtained via the NCBI Gene Expression Omnibus (GEO) website. Different platforms were reconciled using HUGO approved gene symbols, and extracted gene expression data were $\log 2$ transformed, centred and scaled across samples in order to emphasise relative expressions as opposed to absolute values.

\section{Integration of transcriptional and genomic profiles}

Integration of transcriptional and genomic datasets was performed by investigating levels of expression for genes located in regions of copy number variation between groups of interest. Genes whose differences in expression varied in the same direction as differences in copy number between two groups (e.g. relative over-expression of genes in a region of increased copy number) were viewed as genes of interest.

\section{Results}

Molecular and clinical data was available for 69 patients who underwent surgery for NSCLC between May 1999 and July 2006. Demographic and pathologic data are included in table 1. Median follow-up for surviving patients exceeds 35 months. After a median follow-up of 36 months $(1$ - 80) for all patients, 28/68 patients developed recurrent disease, and 23/68 patients died of NSCLC (one patient with disseminated disease at diagnosis excluded from analysis). Comparable to other series of early stage NSCLC [9], five year overall survival rates approximated $55 \%$.

\section{Genomic Analysis \\ Aneuploidy}

All samples demonstrated significant chromosomal instability with an average of 43.6 chromosomal breakpoints per sample (defined by a change in the stepwise copy number along a chromosome), with over 100 and 150 regions of high-level gain (+3) and loss (-3) respectively. There was also a very high rate of low-level genomic alteration (both gains and losses). On average, over 10 whole arm losses or duplications were seen per sample, with a rate of isochromosome formation of 1.6 per genome (duplication of one arm with loss of the opposing arm of the same chromosome). These results are consistent with the highly disordered nature of lung cancer genomes. Comparisons between clinical subgroups of interest revealed remarkably similar degrees of aneuploidy and chromosomal disorder in almost all 
Table 1 Demographic and pathologic details for 69 NSCLC patients

\begin{tabular}{|c|c|c|}
\hline $\begin{array}{l}\text { Variable (number of patients } \\
\text { assessed) }\end{array}$ & Number & $\begin{array}{l}\text { Percentage }(95 \% \\
\mathrm{Cl})\end{array}$ \\
\hline \multicolumn{3}{|l|}{ Gender (69) } \\
\hline Male & 42 & $61(49-72)$ \\
\hline Female & 27 & $39(28-51)$ \\
\hline \multicolumn{3}{|c|}{$\begin{array}{l}\text { Age at definitive treatment (years) } \\
\text { (69) }\end{array}$} \\
\hline Median (range) & $70(29-85)$ & \\
\hline \multicolumn{3}{|l|}{ Histology (69) } \\
\hline$A C$ & 30 & $43(32-55)$ \\
\hline SCC & 23 & $33(23-45)$ \\
\hline LCC & 16 & $23(14-34)$ \\
\hline \multicolumn{3}{|l|}{ Smoking status (69) } \\
\hline Never & 16 & $23(14-34)$ \\
\hline Current/Ex & 53 & $77(66-86)$ \\
\hline \multicolumn{3}{|l|}{ Stage (69) } \\
\hline IA & 16 & $23(14-34)$ \\
\hline IB & 26 & $38(27-49)$ \\
\hline$\| \mathrm{A}$ & 3 & $4(1-11)$ \\
\hline$\| B$ & 7 & $10(5-19)$ \\
\hline$\| \mathrm{A}$ & 8 & $12(6-20)$ \\
\hline$\| \mathrm{B}$ & 3 & $4(1-11)$ \\
\hline IV & $5^{*}$ & $12(6-20)$ \\
\hline Unknown & 1\# & $1(0-7)$ \\
\hline \multicolumn{3}{|l|}{ Primary tumour size $(\mathrm{mm})(69)$} \\
\hline $0-20$ & 23 & $33(23-45)$ \\
\hline $21-30$ & 10 & $15(8-24)$ \\
\hline $31-50$ & 22 & $32(22-43)$ \\
\hline $51-70$ & 5 & $7(3-16)$ \\
\hline$>70$ & 7 & $10(5-20)$ \\
\hline Unknown & $2^{\wedge}$ & $3(0-9)$ \\
\hline T< 40 mm, N1 \&/or M1 (69) & 13 & $19(11-29)$ \\
\hline T > 40 mm, NOMO (69) & 11 & $16(9-26)$ \\
\hline T > 70 mm, NOMO (69) & 4 & $6(2-13)$ \\
\hline$K$-Ras mutation $(46)^{* *}$ & 10 & $21(11-34)$ \\
\hline EGFR mutation $(46)^{* *}$ & 5 & $11(4-22)$ \\
\hline P53 mutation (69) & $26+/-17$ & \\
\hline Definite & 26 & $38(27-49)$ \\
\hline Possible & 17 & $25(16-36)$ \\
\hline Recurrence (68)+ & 28 & $41(30-53)$ \\
\hline Death from NSCLC (68)+ & 23 & $34(23-45)$ \\
\hline \multicolumn{3}{|c|}{$\begin{array}{l}\text { Time from surgery to death (months) } \\
\text { (23) }\end{array}$} \\
\hline Median (range) & $19(7-73)$ & \\
\hline
\end{tabular}

$\mathrm{AC}=$ adenocarcinoma; $\mathrm{LCC}=$ large cell carcinoma; $\mathrm{SCC}=$ squamous cell carcinoma.

*3 patients had solitary cerebral metastases resected at diagnosis, 1 patient had 2 separate lung lesions (unclear if synchronous primary lesions or pulmonary metastasis), 1 patient had disseminated disease.

\# Stage unable to be determined in 1 patient after wedge resection without nodal dissection.

$\wedge$ Tumour size unable to be assessed in 1 patient who underwent incomplete tumour resection after combined chemoradiation, and one who had incomplete resection of an obstructing stage IIIA tumour followed by definitive radiotherapy.

** Only AC and LCC samples were tested for mutations of K-Ras and EGFR. +One patient with disseminated disease at diagnosis excluded from recurrence and survival analysis. groups (Table 2). Specifically, neither prognosis, degree of histologic differentiation, K-Ras or TP53 status was associated with evidence of greater aneuploidy.

\section{Histotype comparisons}

Charts documenting the averaged standardised copy numbers at each BAC location were generated to enable visual comparisons of genomic profiles between AC (23) and SCC (17) (figure 1). Regions of shared change (amplification of 5p15.33-p13.13, loss of 3p26.3-p13) and several regions of difference (SCC: gains at 3q12.1q28 and 12p13.33-p12.1, losses of chromosome 4 (4pter-4qter); AC: gains of 6p25.1-p21.31, losses of 6q13-27, and a greater magnitude of change in several regions of shared loss $(9 p, 13 q, 17 p, 18 q, 19 p, 19 q$ and $22 q)$ or gain $(7 p, 7 q, 8 q))$ were identified. These findings are consistent with previous studies [9-16].

Associations with mutation status in TP53, KRAS and EGFR pathways

Clinical data demonstrated a trend to greater rate of TP53 mutation in SCC than AC (TP53 mutation in 9/12 (75\%) SCC and 9/18 (50\%) AC, p = 0.083). Amplification of $3 q$

Table 2 Comparisons of average number of breakpoints for NSCLC subgroups

\begin{tabular}{|c|c|c|}
\hline Subgroup & $\begin{array}{l}\text { Ave. No. } \\
\text { Breakpoints }\end{array}$ & Ratio of comparison ( $p$ value \\
\hline \multicolumn{3}{|l|}{ Histotype } \\
\hline AC & 45.7 & ACC:SCC = $1.1(1.0)$ \\
\hline SCC & 43.1 & \\
\hline LCC & 39.5 & \\
\hline \multicolumn{3}{|l|}{ Gender } \\
\hline Male (M) & 44.9 & $M: F=1.1(1.0)$ \\
\hline Female (F) & 41.2 & \\
\hline \multicolumn{3}{|l|}{ Smoking } \\
\hline Smokers (S) & 44.5 & $\mathrm{~S}: \mathrm{N}=1.1(1.0)$ \\
\hline Never smokers (N) & 41.1 & \\
\hline \multicolumn{3}{|l|}{ Recurrence } \\
\hline Recurrent (R) & 44.7 & $\mathrm{R}: \mathrm{NR}=1.0(1.0)$ \\
\hline Non-recurrent (NR) & 43.3 & \\
\hline \multicolumn{3}{|l|}{ Survival } \\
\hline Non-survivors (NS) & 46.5 & $\mathrm{NS}: \mathrm{S}=1.1(0.9)$ \\
\hline Survivors (S) & 42.8 & \\
\hline \multicolumn{3}{|l|}{ Metastasis } \\
\hline Metastatic (M) & 40.0 & M:NM = $0.9(0.9)$ \\
\hline Non-metastatic (NM) & 46.8 & \\
\hline \multicolumn{3}{|c|}{ EGFR status } \\
\hline Mutant (m) & 38.6 & $\mathrm{~m}: \mathrm{wt}=0.9(0.9)$ \\
\hline Wild-type (wt) & 44.6 & \\
\hline \multicolumn{3}{|l|}{ K-Ras status } \\
\hline Mutant (m) & 42.3 & $\mathrm{~m}: \mathrm{wt}=1.0(1.0)$ \\
\hline Wild-type (wt) & 43.9 & \\
\hline \multicolumn{3}{|l|}{ p53 status } \\
\hline Mutant (m) & 46.8 & $\mathrm{~m}: \mathrm{wt}=1.1(0.9)$ \\
\hline Wild-type (wt) & 40.8 & \\
\hline
\end{tabular}




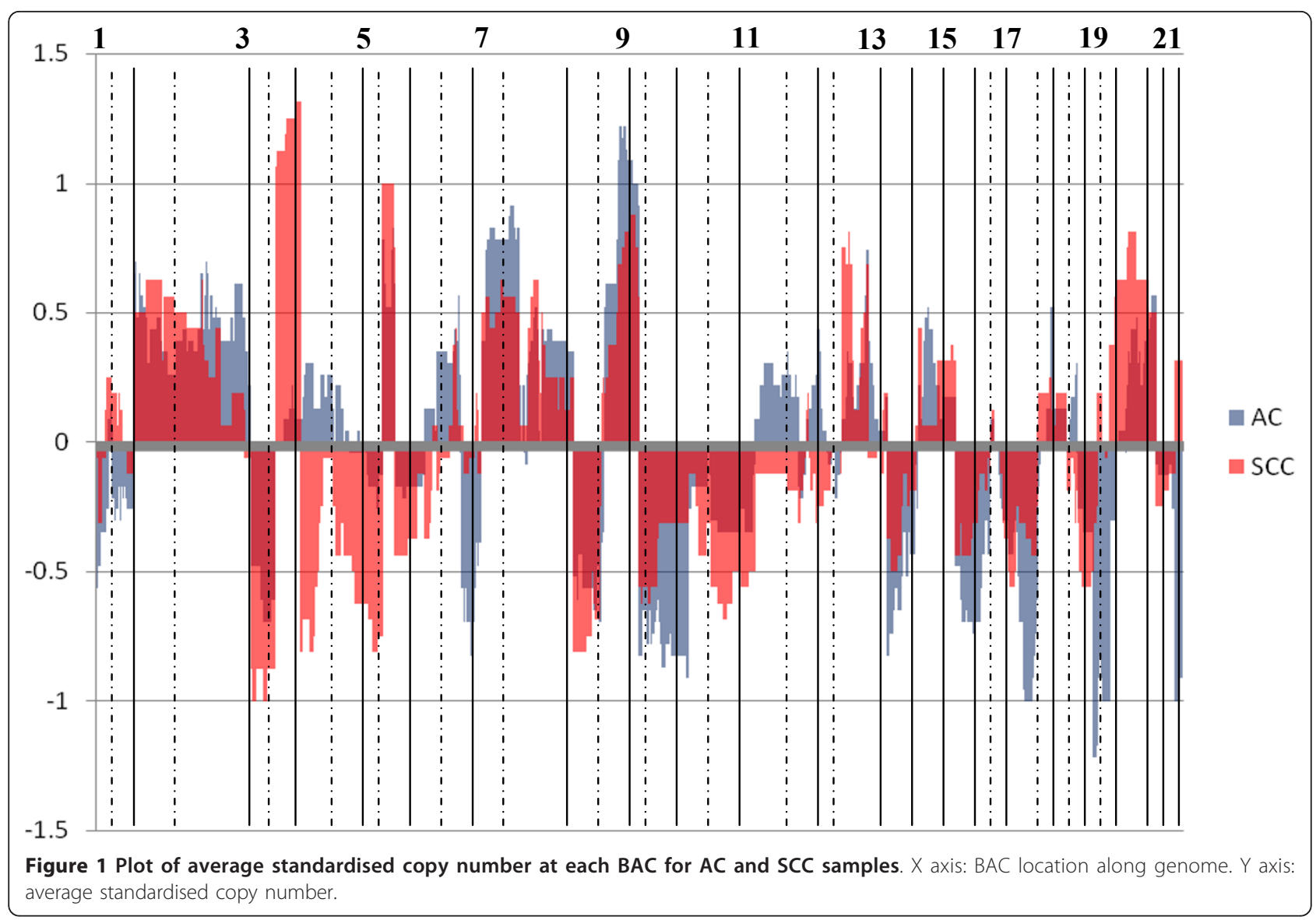

was also more frequent in SCC than AC samples ( $\mathrm{p}=$ 0.004). When analysing all samples, no relationship was found between 3q amplification and TP53 mutation ( $\mathrm{p}=$ 0.99). However, when analysing SCC and AC separately, a statistically significant relationship between TP53 mutation and 3q amplification was detected in AC samples, with amplification of $3 \mathrm{q}$ being significantly more common in TP53 mutant cancers $(1 / 8$ and 4/10 samples with $3 q$ amplification in TP53 wild-type (wt) and mutant AC groups respectively, $\mathrm{p}=0.027$ ). Both TP53 mutant and wt samples more frequently demonstrated copy number loss at the TP53 locus (17p13) than gain (11/18 and 9/12 samples in TP53 mutant and wt groups respectively).

Adenocarcinomas were screened for EGFR and KRAS mutations. Small numbers of EGFR mutant tumours limited detailed analysis. Good quality genomic profiles were available for only 5 tumours with EGFR mutation and no significant differences were seen between the profiles of EGFR mutant and non-mutant tumours.

Genomic profiles were available for 8 KRAS mutant tumours, 31 wt and 16 tumours with unknown KRAS mutation status (untested SCC samples). In averaged copy number charts, KRAS mutant tumours showed a predilection for losses of 1p36.32-p13.2, 6q11.1-q27, 11p13-11q13.2 and 11q21-12p13.1 and gains of 1q21.1q43 (Figure 2).

\section{Associations with metastasis, tumour recurrence and NSCLC-specific survival}

To investigate the notion of inherent metastatic potential, molecular profiles of large $(>4 \mathrm{~cm})$ non-metastatic tumours and small $(<2 \mathrm{~cm})$ metastatic (nodal or distant) tumours were compared. Genomic profiles of 3 'metastatic' and 8 'non-metastatic' tumours revealed some differences in the magnitude of copy number changes, without regions of clear difference between the two groups. There was no correlation between genomic changes and tumour recurrence or survival. There were differences in the magnitude of gene copy number changes at $7 \mathrm{p}, 8 \mathrm{q}, 9 \mathrm{p}, 15 \mathrm{q}$ and $17 \mathrm{p}$ in recurrent compared to non-recurrent tumours. Contained within these regions are the $M Y C$ oncogene (8q), as well as TP53 (17p), and the CDKN2A locus (containing p14(ARF) and p16 tumour suppressor genes (9p) (TSG's)).

\section{Transcriptional Analysis}

\section{Histotype comparisons}

Ranking the genes by moderated $\mathrm{t}$-statistics and selecting a $\mathrm{p}$ value cut-off of $<0.005,310$ genes with differential 
expression between 16 SCC and 25 AC samples were identified, representing the biological processes of cell adhesion, epidermis development, keratinisation and keratinocyte differentiation. A significant proportion of these genes had roles in antigen processing and presentation, and the phosphatidylinositol signalling pathway. Thirty of 310 genes in the differentiating gene list were located on chromosome 3 ( $\mathrm{p}=0.0098)$, implicating genomic changes at this locus in determining NSCLC phenotype. This is consistent with the genomic data, which indicates gain of $3 \mathrm{q}$ is associated with SCC histology.

\section{Associations with mutation status in TP53, K-Ras and EGFR} pathways

Expression levels of 67 genes differed significantly between TP53 mutant (17) and wt (21) tumours. Many of the biological functions represented by these genes were also strongly represented by the genes differentially expressed between AC and SCC. In addition, 20/67 discriminating genes were also included in the gene list differentiating SCC from AC. Hierarchical clustering based on the expression of these 67 differentially expressed genes not only segregated TP53 mutant from wild-type tumours, but also resulted in clustering of SCC samples with the TP53 mutant tumours. Our results suggest that the gene expression signature observed for TP53 mutant tumours may be at least in part related to SCC histology rather than TP53 biology.

Transcriptional profiles of AC and LCC tumours with (8) and without (31) KRAS mutation were compared. Biological processes represented by 108 differentiating genes included cell growth, second-messenger mediated signalling, chromosome organisation and biogenesis, and gene regulation (mediated via histones and their effects on biosynthesis and nucleosome assembly) (table 3). These findings are consistent with other published studies linking KRAS mutation to increased translation of cancer related proteins, and chromosome instability $[17,18]$. The low frequency of EGFR mutant cancers precluded statistically meaningful analysis of transcriptional data according to EGFR genotype.

\section{Associations with metastasis, tumour recurrence and NSCLC-specific survival}

Transcriptional profiles identified 39 genes that differentiated between 19 'metastatic' and 35 'non-metastatic' tumours, with molecular pathways involved in protein translation most strongly represented (MRPL33, RPL12, RPL27A, RPS5, RPS9). Comparison of expression profiles of 14 tumours recurring within 12 months of surgery to remaining samples identified 60 genes with differential expression between the two groups, with a 
Table 3 Details of genes over-expressed in K-Ras mutant tumours

\begin{tabular}{|c|c|c|c|c|}
\hline Symbol & Gene Name & Cytoband & $P$ value & Gene Function \\
\hline ACOX2 & Acyl-Coenzyme A oxidase 2, branched chain & $3 p 14.3$ & $4.9 \mathrm{E}^{-3}$ & Lipid metabolism \\
\hline ALDH2 & Aldehyde dehydrogenase 2 family (mitochondrial) & $12 q 24.2$ & $9.8 E^{-4}$ & Alcohol metabolism \\
\hline ALDH3B1 & Aldehyde dehydrogenase 3 family, member B1 & $11 q 13$ & $3.9 E^{-3}$ & Alcohol metabolism \\
\hline AREG & Amphiregulin (schwannoma-derived growth factor) & $4 q 13-q 21$ & $1.4 \mathrm{E}^{-4}$ & Autocrine growth factor family member \\
\hline CD55 & $\begin{array}{l}\text { CD55 molecule, decay accelerating factor for } \\
\text { complement (Cromer blood group) }\end{array}$ & $1 \mathrm{q} 32$ & $6.9 E^{-4}$ & $\begin{array}{l}\text { Immune response, protection of cells from } \\
\text { complement mediated damage }\end{array}$ \\
\hline CLDN10 & Claudin 10 & $\begin{array}{l}13 q 31- \\
q 34\end{array}$ & $2.9 E^{-3}$ & Intercellular tight junctions \\
\hline DGKD & Diacylglycerol kinase, delta $130 \mathrm{kDa}$ & $2 q 37.1$ & $1.0 E^{-3}$ & Intercellular signalling, cell growth. \\
\hline EGR1 & Early growth response 1 & $5 q 31.1$ & $2.5 E^{-3}$ & $\begin{array}{l}\text { Transcriptional regulation of genes involved in } \\
\text { mitogenesis }\end{array}$ \\
\hline MSLN & Mesothelin & $16 p 13.3$ & $4.61 \mathrm{E}^{-5}$ & Possible role in cell adhesion \\
\hline MST1R & $\begin{array}{l}\text { Macrophage stimulating } 1 \text { receptor (c-met-related } \\
\text { tyrosine kinase) }\end{array}$ & $3 p 21.3$ & $4.5 \mathrm{E}^{-3}$ & Cell motility, positive regulation of cell cycle \\
\hline NPC2 & Niemann-Pick disease, type C2 & $14 q 24.3$ & $4.6 E^{-4}$ & Regulation of cholesterol transport and storage \\
\hline NR4A1 & Nuclear receptor subfamily 4, group A, member 1 & $12 q 13$ & $6.2 \mathrm{E}^{-4}$ & Transcription factor \\
\hline PFDN1 & Prefoldin subunit 1 & $5 q 31$ & $1.5 E^{-3}$ & Cell cycle, transcription factor activity, protein folding \\
\hline PTGS2 & $\begin{array}{l}\text { Prostaglandin-endoperoxide synthase } 2 \text { (cyclo-oxygenase } \\
\text { 2) }\end{array}$ & $\begin{array}{l}1 \mathrm{q} 25.2- \\
\mathrm{q} 25.3\end{array}$ & $3.1 \mathrm{E}^{-3}$ & Prostaglandin biosynthesis \\
\hline ST3GAL5 & ST3 beta-galactoside alpha-2,3-sialyltransferase 5 & $2 p 11.2$ & $1.4 \mathrm{E}^{-3}$ & $\begin{array}{l}\text { Cell differentiation, cell proliferation, signal } \\
\text { transduction. }\end{array}$ \\
\hline TFPI2 & Tissue factor pathway inhibitor 2 & $7 q 22$ & $4.5 E^{-4}$ & Matrix remodelling, coagulation \\
\hline UPP1 & Uridine phosphorylase 1 & $7 p 12.3$ & $1.0 E^{-4}$ & Nucleotide catabolism \\
\hline
\end{tabular}

common theme of RAS activation represented in ontological and single gene analyses. Included in the differentiating gene list were MAPK1, DUSP11 and DUSP13, PTPN11, and PIK3CB, all having roles in signal transduction and the MAPK pathway. The phosphatidylinositol signalling pathway was also significantly over-represented in ontology analysis.

Expression levels of only 38 genes differed significantly between deceased and surviving patients. 18 of these genes were shared with gene lists of recurrent vs. nonrecurrent tumours. Few biological processes were represented by more than one gene, and clear patterns of gene ontology were not apparent.

\section{Correlation with External Data Sets}

Comparison of our differential gene list for recurrence with the discriminating gene list for survival in GSE11117 (transcriptional and survival data for 41 NSCLC samples, using Novachip Human 34.5k microarray interrogating 34,500 transcripts for each sample; http://www.ncbi.nlm.nih.gov/ geo/query/acc.cgi?acc=GSE11117) identified 40 matched transcripts (additional file 5). Log-transformed expression values of the 40 transcripts were used to classify the samples from GSE11117 into two subgroups using a correlation, average-linkage hierarchical clustering $\mathrm{R}$ package. Kaplan-Meier curves for these external samples using the 40 transcripts matched to our recurrence gene list demonstrated statistically significant survival prediction (figure 3, $\mathrm{p}<0.0153)$ ), with 21 and 20 samples in each group.

\section{Integration of Genomic and Transcriptional Profiles}

To determine whether integration of genomic and expression data added to the predictive value of these datasets for histologic classification, we identified 34 genes whose copy number and expression varied between $\mathrm{AC}$ and SCC, 24 of which demonstrated concordant differences in copy number and expression (table 4). Notably, 17 of 24 differentiating genes were located on chromosome $3 \mathrm{q}$.

\section{Associations with mutation status}

Examination of transcriptional data from 1p, 1q, 6q, $11 \mathrm{p}, 11 \mathrm{q}$ and $12 \mathrm{p}$ (regions of genomic difference) identified 25 genes with concordant differences in copy number and expression between $K$-Ras mutant and wt tumours (table 5). A number of genes demonstrated reduced copy number and expression in KRAS mutant tumours, including putative tumour suppressor genes (FOXO3, EXTL2, PPP2R1B), negative regulators of the receptor tyrosine kinase oncogenic pathways (PTPRK, $D G K Z$, NCAM1), and negative regulators of Ras (EPHB2). Several over-expressed genes located in regions of amplification play roles in constitutive KRAS activation (PTGS2/COX2), enhanced transactivation of the EGFR (RGS2), enhanced invasive potential (ECM1), and MAPK/ERK activation (PTGS2/COX2).

\section{Associations with metastasis, tumour recurrence and} NSCLC-specific survival

Investigation of genomic and transcriptional data identified only 2 genes (ARFGEF1 and PENK) whose copy number and expression differentiated 'metastatic' from 

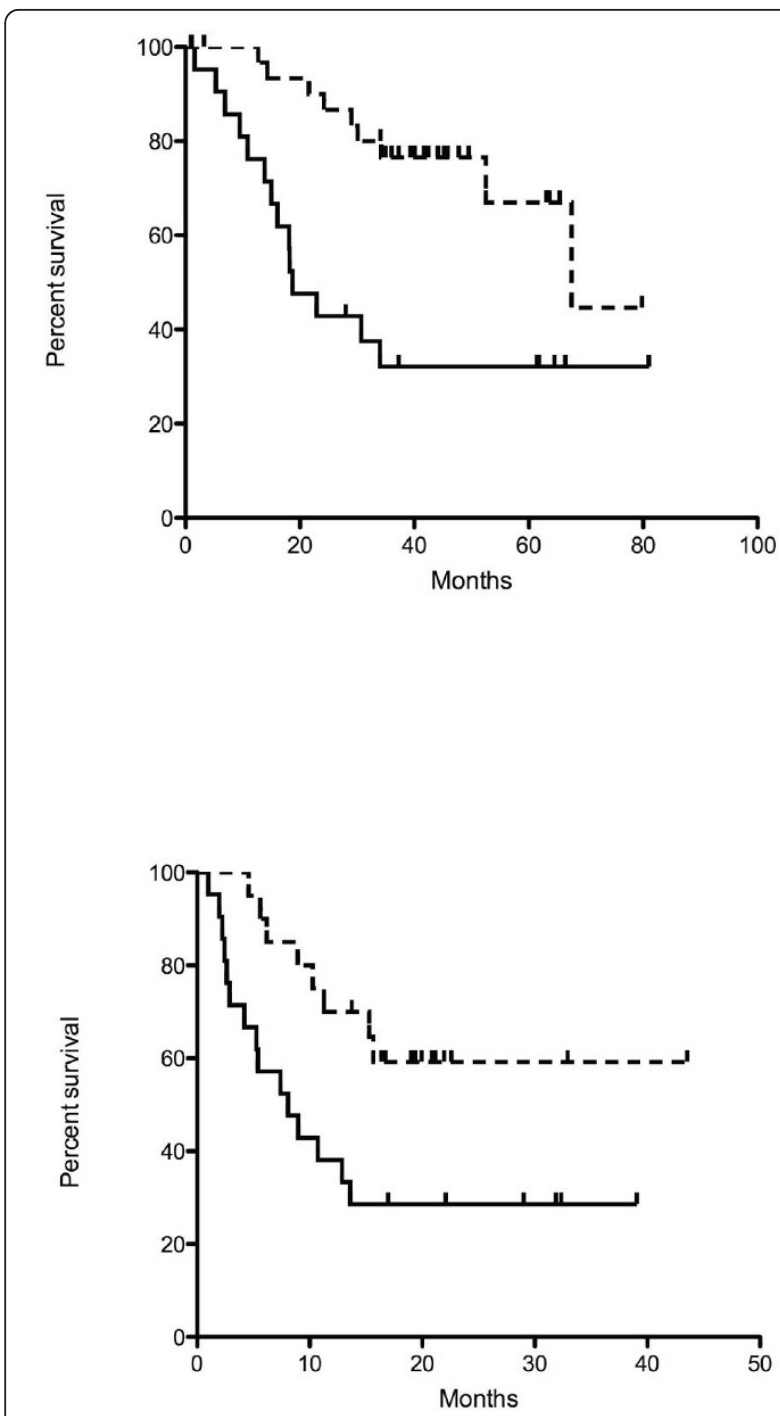

Figure 3 Survival curves based on recurrence differential gene list. A. Recurrence free survival curve for our dataset grouped by recurrence differential gene list. B: Kaplan Meier survival curve for external dataset GSE11117 using 40 transcripts matched to our recurrence differential gene list to classify.

'non-metastatic' tumours, neither of which have been previously implicated in malignancy. Similarly, correlation of transcriptional and genomic data identified only 3 genes with concordant differences in recurrence and survival comparisons - SMARCA2, MINK and RECK.

\section{Discussion}

The clinical, demographic and pathologic characteristics of this NSCLC cohort are consistent with the published literature. The transcriptional and genomic profiles identified in this study should therefore be generalisable to other patients with early-stage NSCLC. The tumour samples analysed demonstrated substantial genomic instability, with comparisons between subgroups failing to demonstrate any significant difference. Previous studies of copy number changes in NSCLC have found no association between age, gender, histology, stage or tumour grade and the degree of genomic instability [19-21]. The absence of difference in the degree of genomic abnormalities between KRAS mutant and wt tumours is interesting, as both our transcriptional and genomic data imply enhanced activity of genes involved in chromosome structure and organisation in KRAS mutant tumours. We recognise that there were a small number of KRAS mutant tumours available for comparison and this may have limited our analysis.

Consistent with previously reported studies [9-16], the major differences in copy number and gene expression profiles between AC and SCC of the lung involved chromosome 3q. The strong independent correlation with amplification and over-expression at this locus suggests a causal relationship in SCC for genes in this region which warrant further investigation. These include $T P 73 L$, a gene extensively implicated in SCC, whose expression was most strongly correlated with the SCC phenotype, and which has been previously reported to be a putative oncogene [22-32]. While the role of TP73L in squamous cell pathogenesis remains unclear, a recent study of SiRNA mediated TP73L inhibition in SCC resulted in reduced cell survival with maintenance of squamous characteristics [28]. These results suggest that TP73L is important in SCC cell survival. Other genes previously shown to be over-expressed in SCC were included in our differentiating list (CSTA [33,34], FGFBP1), and warrant functional validation.

The differential copy number and expression levels between AC and SCC of TNFSF10/TRAIL and ABCC5, which have roles in apoptosis and chemoresistance respectively, may have implications for treatment of NSCLC. Recently published clinical data [35] suggest there are histotype-specific differences in response to systemic therapies. Validation of the differential activity of the roles of these genes and sensitivity to conventional and novel chemotherapeutic agents may be an area for future research.

Published data on the relationship between TP53 mutations and histotype in NSCLC is conflicting [36,37]. SCC were associated with more frequent TP53 mutations than $\mathrm{AC}$ in our dataset. Cigarette smoking is a causal factor for both SCC phenotype and TP53 mutation [37]. However, we also observed a correlation between 3q amplification and TP53 mutations in AC samples. This suggests that the apparent association between TP53 mutations and SCC may be mediated by the relationship between TP53 mutations and amplification of regions of 3q. We caution that this study is underpowered to draw strong conclusions regarding the role of TP53 in NSCLC pathogenesis.

Tumours possessing mutations of KRAS express genes playing key roles in cell growth, chromosome organisation 
Table 4 Details of genes located in regions of genomic difference with concordant differences in expression levels between AC and SCC

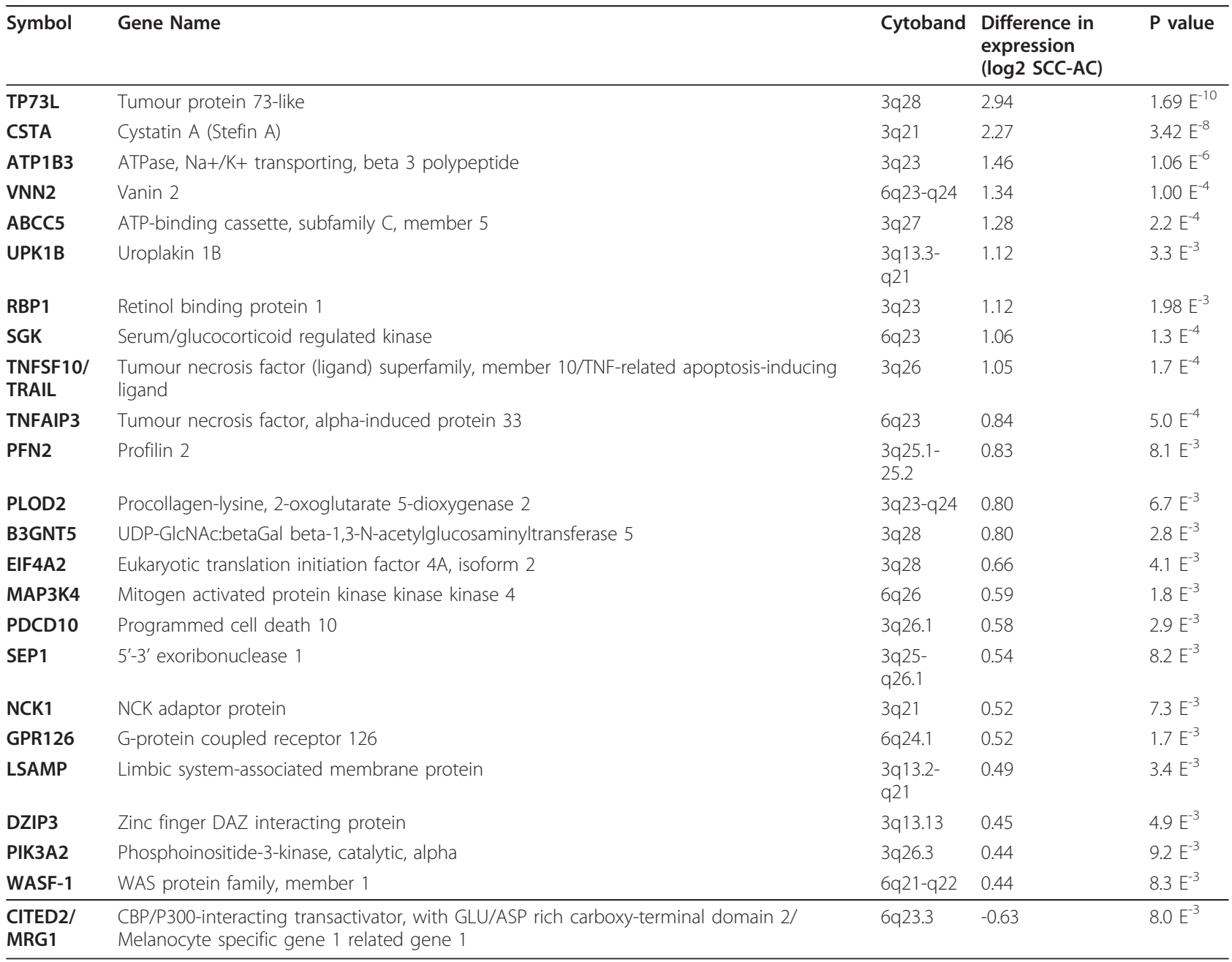

and gene regulation. As previously reported, we identified amplification and over-expression of COX2 in KRAS mutant tumours. KRAS mutant tumours did not demonstrate mutations in EGFR consistent with previous reports in both NSCLC and colon carcinoma which suggest that KRAS mutations predict resistance to EGFR antagonists [29,38-41]. Several reports link NCAM1 to Ras-dependent activation of ERK MAPK's [42,43]. Reduced copy number and expression of NCAM1 in tumours bearing KRAS mutations, as seen in our data, has not previously been reported. Further research into a KRAS mutation profile may yield simple and reliable immunohistochemical markers of KRAS mutation, thereby significantly reduce the cost of determining $K R A S$ status in clinical practice.

The gene expression profile observed in 'metastatic' tumours is consistent with a growing body of literature implicating deregulated protein synthesis in the development and metastatic potential of human cancers $[44,45]$. Increased mRNA translation is a critical downstream function of many cancer related genes, and many gene products with roles in metastasis are not mutated but inappropriately expressed in malignant cells (e.g. VEGF, c-Myc, fos, Her2Neu, PDGF) [18]. Opportunities for therapeutic intervention currently in development include oncolytic viruses that require deregulated protein translation for their replication [18], or agents that inhibit mTOR, an integral factor in protein translation (eg. temsirolimus (CCI-779), everolimus (RAD001) and deforolimus (AP23573)).

While the small number of recurrences and deaths due to NSCLC in our tumour-set makes it difficult to draw strong conclusions, transcriptional profiles linked to tumour recurrence suggest KRAS pathway activation. This may be due to a higher proportion of AC and LCC vs. SCC in the 'recurrent' group. Other regions of copy number change demonstrate genomic gains in the region of $c-M y c$ and losses in the region of $p 16$ (INK4a, $C D N K 2 A)$ in recurrent or non-survivor tumours, supporting a prognostic association of the $M y c: C D N K 2 A$ 
Table 5 Details of genes with differential copy number and expression between $K$-Ras mutant and wild-type tumours

\begin{tabular}{|c|c|c|c|c|}
\hline Symbol & Gene name & Cytoband & $\begin{array}{l}\text { Difference in } \\
\text { expression } \\
\text { (log2 mutant - wild } \\
\text { type) }\end{array}$ & $P$ value \\
\hline RGS2 & Regulator of G-protein signaling 2, 24kDa & $1 \mathrm{q} 31$ & 1.99 & $5.9 E^{-3}$ \\
\hline NCAM1 & Neural cell adhesion molecule 1 & $\begin{array}{l}11 \text { ( multiple } \\
\text { clusters) }\end{array}$ & -1.63 & $1.5 E^{-3}$ \\
\hline CD55 & CD55 molecule, decay accelerating factor for complement (Cromer blood group) & $1 \mathrm{q} 32$ & 1.49 & $6.9 \mathrm{E}^{-4}$ \\
\hline $\begin{array}{l}\text { PTGS2/ } \\
\text { COX-2 }\end{array}$ & $\begin{array}{l}\text { Prostaglandin-endoperoxide synthase } 2 \text { (prostaglandin G/H synthase and } \\
\text { cyclooxygenase)/Cyclooxygenase- } 2\end{array}$ & $1 \mathrm{q} 25.2-\mathrm{q} 25.3$ & 1.32 & $3.1 \mathrm{E}^{-3}$ \\
\hline ECM1 & Extracellular matrix protein 1 & $1 \mathrm{q} 21$ & 1.21 & $5.9 \mathrm{E}^{-3}$ \\
\hline EXTL2 & Exostoses (multiple)-like 2 & $1 \mathrm{p} 21$ & -1.08 & $4.2 E^{-3}$ \\
\hline HIST2H2BE & Histone cluster 2, H2be & $1 q 21-q 23$ & 1.07 & $2.7 E^{-3}$ \\
\hline H2AA & Histone 2 & $1 q 21$ & 0.95 & $1.2 \mathrm{E}^{-3}$ \\
\hline CHEK1 & CHK1 checkpoint homolog (S. pombe) & $11 q 24-q 24$ & -0.94 & $6.1 \mathrm{E}^{-3}$ \\
\hline GPX7 & Glutathione peroxidase 7 & $1 \mathrm{p} 32$ & -0.88 & $5.6 E^{-3}$ \\
\hline RWDD2A & RWD domain containing $2 \mathrm{~A}$ & $6 q 14.2$ & -0.80 & $8.7 E^{-4}$ \\
\hline HSF2 & Heat shock transcription factor 2 & $6 q 22.31$ & -0.78 & $1.6 \mathrm{E}^{-3}$ \\
\hline BLR1 & $\begin{array}{l}\text { Burkitt lymphomas receptor 1, GTP binding protein (chemokine (CXC motif) } \\
\text { receptor 5) }\end{array}$ & $11 \mathrm{q} 23.3$ & -0.69 & $7.4 E^{-3}$ \\
\hline MIZF & MBD2-interacting zinc finger & $11 q 23.3$ & -0.69 & $5.9 E^{-3}$ \\
\hline KPNA5 & Karyopherin alpha 5 (importin alpha 6) & $6 q 22.2$ & -0.66 & $8.7 \mathrm{E}^{-3}$ \\
\hline LMO4 & LIM domain only 4 & $1 \mathrm{p} 22.3$ & -0.66 & $3.2 E^{-3}$ \\
\hline MUTYH & MutY homolog (E. coli) & 1 p34.3-p32.1 & -0.65 & $4.9 E^{-4}$ \\
\hline REV3L & REV3-like, catalytic subunit of DNA polymerase zeta (yeast) & $6 q 21$ & -0.65 & $8.8 \mathrm{E}^{-3}$ \\
\hline EPHB2 & Ephrin B2 & 1 p36.1-p35 & -0.64 & $6.7 E^{-3}$ \\
\hline DGKZ & Diacylglycerol kinase, zeta $104 \mathrm{kDa}$ & $11 p 11.2$ & -0.64 & $9.2 E^{-3}$ \\
\hline TSPYL4 & TSPY-like 4 & $6 \mathrm{q} 22.1$ & -0.63 & $1.6 E^{-3}$ \\
\hline DBT & Dihydrolipoamide branched chain transacylase E2 & $1 \mathrm{p} 31$ & -0.58 & $5.8 \mathrm{E}^{-3}$ \\
\hline FOXO3 & Forkhead box O3 & $6 q 21$ & -0.53 & $5.7 \mathrm{E}^{-3}$ \\
\hline PPP2R1B & Protein phosphatase 2 , regulatory subunit $A$, beta isoform & $11 q 23.2$ & -0.51 & $2.7 E^{-3}$ \\
\hline PTPRK & Protein tyrosine phosphatase, receptor type, kappa & $6 q 22.2-q 22.3$ & -0.08 & $4.3 E^{-4}$ \\
\hline
\end{tabular}

ratio in NSCLC, as has been described in head and neck SCC [46]. Specific genes linked to recurrence or survival include SMARCA2 (implicated in the regulation of gene expression cell cycle control and oncogenesis), MINK (linked to the JNK MAP kinase pathway) [47] and $R E C K$, which has putative roles in the suppression of tumour growth, invasion, angiogenesis and metastasis [48]. KRAS mutation has been associated with reduced expression of RECK in NSCLC [49], consistent with the clinical observation of poor outcome in patients with KRAS mutation bearing NSCLC. Activation of the Ras pathway may reduce $R E C K$ expression and thereby increase tumour recurrence. Importantly, our prognostic gene signature was validated in an independent test set, suggesting that these findings may eventually yield prognostic markers in resected early-stage NSCLC to better select patients for adjuvant treatments.

\section{Conclusions}

Several molecular alterations have been identified in association with NSCLC histotype, KRAS mutation,
TP53 mutation, metastatic potential, disease recurrence and survival. Although the size of the current study is small, our findings are in many cases consistent with those of previous studies, and have been validated in the case of the prognostic classifier in an independent test set. In addition, several novel molecular changes associated with clinically relevant endpoints have been demonstrated. It is hoped that these results will contribute to identifying new predictive markers and targets for novel therapies to improve treatment selection and better outcomes for patients with this deadly disease.

\section{Additional material}

Additional file 1: Protocol for extraction of RNA

Additional file 2: Protocols for extraction of DNA

Additional file 3: Transcriptional profiling

Additional file 4: Genomic profiling

Additional file 5: 40 matched transcripts between GSE11117 and our differential gene list for recurrence 


\section{Acknowledgements}

The authors would like to acknowledge the assistance of Michael Krypuy from the Department of Pathology, Peter MacCallum Cancer Centre in undertaking high resolution melting analysis for KRAS mutations. Funding for this project was received from The University of Melbourne (scholarship for Dr G Newnham), the Australasian Society of Cardiac and Thoracic Surgeons Foundation, and The National Health and Medical Research Council of Australia.

\section{Author details}

'Department of Oncology, St Vincent's Hospital, (Victoria Pde), Melbourne, (3065), Australia. ²Department of Respiratory Medicine, St Vincent's Hospital, (Victoria Pde), Melbourne, (3065), Australia. ${ }^{3}$ Department of Pathology, Peter MacCallum Cancer Centre, (St Andrews Place), East Melbourne, (3002), Australia. ${ }^{4}$ Department of Anatomical Pathology, St Vincent's Hospital, (Victoria Pde), Melbourne, (3065), Australia. ${ }^{5}$ Bioinformatics Core Facility, Peter MacCallum Cancer Centre, (St Andrews Place), East Melbourne, (3002), Australia. ${ }^{6}$ Department of Thoracic Surgery, St Vincent's Hospital, (Victoria Pde), Melbourne, (3065), Australia. ${ }^{7}$ Centre for Genomics and Predictive Medicine, Peter MacCallum Cancer Centre, (St Andrews Place), East Melbourne, (3002), Australia. ${ }^{8}$ Department of Medicine, St Vincent's Hospital The University of Melbourne, (Tin Alley), Melbourne, (3010), Australia. ${ }^{9}$ Department of Pathology, The University of Melbourne, (Tin Alley), Melbourne, (3010), Australia.

\section{Authors' contributions}

GN performed DNA and RNA extraction and transcriptional microarray studies, and drafted the manuscript. MC, SM and GW participated in the design of the study and co-ordinated collection of clinical data. AD performed and supervised mutation analyses. HD performed mutational analyses. $J \mathrm{~L}$ and NT performed statistical analysis. KO performed pathology review. DT and MC conceived the idea of the study. DT supervised molecular studies and assisted in the preparation of the manuscript. All authors read and approved the final copy of the manuscript.

\section{Competing interests}

The authors declare that they have no competing interests.

Received: 23 August 2010 Accepted: 7 March 2011

Published: 7 March 2011

\section{References}

1. Maione $P$, et al: Combining targeted therapies and drugs with multiple targets in the treatment of NSCLC. Oncologist 2006, 11:274-84, 3.

2. Morgan T, et al: Molecular profiling of Giant Cell Tumour of Bone and Osteoclastic Localization of Ligand for Receptor Activator of Nuclear Factor kB. American Journal of Pathology 2005, 167:117-28.

3. Weiss $M M$, et al: Genomic alterations in primary gastric adenocarcinomas correlate with clinicopathological characteristics and survival. Cell Oncol 2004, 26:307-17, 5-6.

4. Krypuy $M_{1}$, et al: High resolution melting for mutation scanning of TP53 exons 5-8. BMC Cancer 2007, 7, 168 PMID 17764544.

5. Krypuy $M$, et al: High resolution melting analysis for the rapid and sensitive detection of mutations in clinical samples: KRAS codon 12 and 13 mutations in non-small cell lung cancer. BMC Cancer 2006, 6, 295 PMID 17184525

6. Smyth G: Linear models and empirical Bayes methods for assessing differential expression in microarray experiments. Statistical applications in genetics and molecular biology 2004, Article 3.

7. Smyth $\mathrm{G}$, et al: The use of with-in array replicate spots for assessing expression in microarray experiments. Bioinformatics 2005, 2067.

8. Olshen $A$, et al: Circular binary segmentation for the analysis of arraybased DNA copy number data. Biostatistics 2004, 5:557-72

9. Petersen I, et al: Patterns of chromosomal imbalances in adenocarcinoma and squamous cell carcinoma of the lung. Cancer Res 1997, 57:2331-5, 12.

10. Bjorkqvist $A$, et al: DNA gains in $3 q$ occur frequently in squamous cell carcinoma of the lung, but not in adenocarcinoma. Genes Chromosomes Cancer 1998, 22:79-82, 1
11. Pei J, et al: Genomic imbalances in human lung adenocarcinomas and squamous cell carcinomas. Genes Chromosomes Cancer 2001, 31:282-87, 3

12. Massion P, et al: Genomic copy number analysis of non-small cell lung cancer using array comparative genomic hybridisation: implications of the phosphatidylinositol 3-kinase pathway. Cancer Res 2002, 62:3636-40, 13.

13. Massion $P$, et al: Early involvement of the phosphatidylinositol 3-kinase/ Akt pathway in lung cancer progression. Am J Respir Crit Care Med 2004 170:1088-94, 10

14. Kim T, et al: Genome-wide screening of genomic alterations and their clinicopathologic implications in non-small cell lung cancers. Clin Cancer Res 2005, 11:8235-42, 23.

15. Tonon $\mathrm{G}$, et al: High-resolution genomic profiles of human lung cancer. Proc Nat Ac Science USA 2005, 9625-30.

16. Garnis $C$, et al: High resolution analysis of non-small cell lung cancer cell lines by whole genome tiling path array CGH. Int J Cancer 2006 118:1556-64.

17. Graff J, Zimmer S: Translational control and metastatic progression: enhanced activity of the mRNA cap-binding protein elF-4E selectively enhances translation of metastasis-related mRNAs. Clinical and Experimental Metastasis 2003, 20:265-73.

18. Bilanges B, Stokoe D: Mechanisms of translational deregulation in human tumors and therapeutic intervention strategies. Oncogene 2007 5973-5990.

19. Sahin AA, et al: Flow cytometric analysis of the DNA content of nonsmall cell lung cancer. Ploidy as a significant prognostic indicator in squamous cell carcinoma of the lung. Cancer 1990, 65:530-7, 3.

20. Granone $P$, et al: DNA flow cytometric analysis in patients with operable non-small cell lung carcinoma. Eur J Cardiothorac Surg 1993, 7:351-5, 7 .

21. Kasprzyk M, et al: Prognostic value of DNA ploidy: 5-year follow-up of patients with resectable squamous cell carcinoma (SCC) of the lung. Lung Cancer 2006, 51:201-6, 2.

22. Choi $H$, et al: Differential expression of p53 gene family members p63 and p73 in head and neck squamous tumorigenesis. Human Pathology 2002, 33:158-64, 2

23. Barbieri C, Barton C, Pietenpol J: Delta Np63 alpha expression is regulated by the phosphoinositide 3-kinase pathway. The journal of biological chemistry 2003, 51:51408-14.

24. Glickman J, et al: Expression of p53-related protein p63 in the gastrointestinal tract and in esophageal metaplastic and neoplastic disorders. Human Pathology 2001, 32:1157-65, 11.

25. Di Como C, et al: p63 expression profiles in human normal and tumour tissues. Clin Cancer Res 2002, 8:494-501, 2

26. Pruneri $G$, et al: $p 63$ in laryngeal squamous cell carcinoma: evidence for a role of TA-p63 down-regulation in tumorigenesis and lack of prognostic implications of p63 immunoreactivity. Lab Invest 2002, 82:1327-34, 10

27. Massion $P$, et al: Significance of p63 amplification and overexpression in lung cancer development and prognosis. Cancer Res 2003, 63:7113-21, 21.

28. Au N, et al: P63 expression in lung carcinoma: a tissue microarray study of 408 cases. Appl Immunohistochem Mol Morphol 2004, 12:240-47, 3.

29. Van Cutsem E, Nowacki M, Lang I: Randomized phase III study of irinotecan and 5FU/FA with or without cetuximab in the first-line treatment of patients with metastatic colorectal cancer: The CRYSTAL trial. J Clin Pathol 2007, 25, 164s abstr 4000.

30. Thurfell $\mathrm{N}$, et al: Complex p63 mRNA isoform expression patterns in squamous cell carcinoma of the head and neck. Int J Oncol 2004, 25:27-35, 1.

31. Nylander $K$, et al: Differential expression of p63 isoforms in normal tissues and neoplastic cells. J Pathol 2002, 198:417-27, 4

32. De Young MP, et al: Tumor-specific p73 up-regulation mediates p63 dependence in squamous cell carcinoma. Cancer Res 2006, 66:9362-8, 19.

33. Ebert $E$, et al: Expression of cysteine protease inhibitors stefin A, stefin B, and cystatin C in human lung tumor tissue. Adv Exp Med Biol 1997, 421:259-65.

34. Werle B, et al: Cystatins in non-small cell lung cancer: tissue levels, localization and relation to prognosis. Oncol Rep 2006, 16:647-55, 4.

35. Scagliotti GV, et al: Phase III study comparing cisplatin plus gemcitabine with cisplatin plus pemetrexed in chemotherapy-naive patients with 
advanced-stage non-small-cell lung cancer. J Clin Oncol 2008, 26:3543-51, 21.

36. Takahashi T, et al: p53: a frequent target for genetic abnormalities in lung cancer. Science 1989, 246:491-4, 4929.

37. Sekido Y, Fong K, Minna J: Molecular biology of lung cancer. [book auth.] V DeVita, S Hellman and S Rosenberg. In Cancer - principles and practice of oncology. s.l. Volume 1. Lippincott Williams \& Wilkins; 2001:917-25.

38. Pao W, et al: KRAS mutations and primary resistance of lung adenocarcinomas to gefitinib or erlotinib. PloS Med 2005, 2:c73, 3 .

39. Peeters $M$, et al: Association of progression-free survival, overall survival, and patient-reported outcomes by skin toxicity and KRAS status in patients receiving panitumumab monotherapy. Cancer 2009, 115:1544-54, 7.

40. Bokemeyer $\mathrm{C}$, et al: Fluorouracil, leucovorin, and oxaliplatin with and without cetuximab in the first-line treatment of metastatic colorectal cancer. J Clin Oncol 2009, 27:663-71, 5.

41. Amado RG, et al: Wild-type KRAS is required for panitumumab efficacy in patients with metastatic colorectal cancer. J Clin Oncol 2008, 26:1626-34, 10.

42. Schmid RS, et al: NCAM stimulates the Ras-MAPK pathway and CREB phosphorylation in neuronal cells. J Neurobiol 1999, 38:542-58, 4.

43. Son H, et al: Reciprocal actions of NCAM and tPA via a Ras-dependent MAPK activation in rat hippocampal neurons. Biochem Biophys Res Commun 2002, 298:262-8, 2.

44. Ramaswamy $\mathrm{S}$, et al: A molecular signature of metastasis in primary solid tumours. Nature Genetics 2003, 33:49-54

45. Weigelt $B$, et al: Gene expression profiles of primary breast tumours maintained in distant metastases. Proc Nat Acad Sciences USA 2003, 100:15901-05, 26.

46. Akervall J, et al: The gene ratios c-MYC:cyclin-dependent kinase (CDK) N2A and CCND1:CDKN2A correlate with poor prognosis in squamous cell carcinoma of the head and neck. Clin Cancer Res 2003, 9:1750-5, 5.

47. Hu Y, et al: Identification and functional characterization of a novel human Misshapen/Nck interacting kinase-related kinase, hMINK-beta. J Biol Chem 2004, 54387-54397.

48. Oh J, et al: The membrane-anchored MMP inhibitor RECK is a key regulator of extracellular matrix integrity and angiogenesis. Cell 2001, 107:789-800.

49. Chang HC, Cho CY, Hung WC: Downregulation of RECK by promoter methylation correlates with lymph node metastasis in non-small cell lung cancer. Cancer Sci 2007, 98:169-73, 2

\section{Pre-publication history}

The pre-publication history for this paper can be accessed here: http://www.biomedcentral.com/1471-2407/11/93/prepub

doi:10.1186/1471-2407-11-93

Cite this article as: Newnham et al: Integrated mutation, copy number and expression profiling in resectable non-small cell lung cancer. $B M C$ Cancer 2011 11:93.

\section{Submit your next manuscript to BioMed Central and take full advantage of:}

- Convenient online submission

- Thorough peer review

- No space constraints or color figure charges

- Immediate publication on acceptance

- Inclusion in PubMed, CAS, Scopus and Google Scholar

- Research which is freely available for redistribution 